\title{
SEQUENTIAL CONVERGENCE IN THE ORDER DUALS OF CERTAIN CLASSES OF RIESZ SPACES
}

\author{
BY
}

P. G. DODDS

\begin{abstract}
Several results of Hahn-Vitali-Saks type are given for sequences in the order dual of an Archimedean Riesz space with separating order dual. The class of Riesz spaces considered contains those which are Dedekind $\sigma$ complete, or have the projection property or have an interpolation property introduced by G. L. Seever. The results depend on recent work of O. Burkinshaw and some results of uniform boundedness type.
\end{abstract}

0. Introduction. A principal aim of this paper is to place in perspective, via the theory of Riesz spaces, a number of classical results concerning sequences of countably additive measures, along with some of their various extensions to finitely additive measures. The results referred to are the well-known convergence theorem of Vitali-Hahn-Saks [14], [17] and the boundedness theorem of Nikodym [13]. Of the more recent extensions of these theorems to finitely additive measures, we wish to mention explicitly the papers of Andô [3], Seever [15] and Wells [16]. A partially ordered set $(P, \lessgtr)$ is said to have property I iff for any sequences $\left\{x_{n}\right\}_{n \in \omega},\left\{y_{m}\right\}_{m \in \omega}$ in $P$ with $x_{n} \leqslant y_{m}$ for all $n, m$, there exists $x \in P$ such that $x_{n} \leqslant x \leqslant y_{n}$ for every $n$. In [15], the Vitali-Hahn-Saks and Nikodym theorems are extended to finitely additive measures defined on a Boolean algebra with property I. That property I plays an important role is further emphasized in [16] and our debt to this paper should be acknowledged. Closely related to these results [15] is that for sequences in the Banach dual of $C(X)$, the space of real-valued continuous functions on the compact Hausdorff space $X$, the notions of weak and weak* convergence coincide, provided that the Riesz space $C(X)$ has property I. This result is due to Grothendieck [8] if $X$ is Stonian and Andô [3] if $X$ is $\sigma$-Stonian. It is of interest then to point out that there are connected spaces $X$ for which $C(X)$ has property I. One such example is $\beta \mathbf{R}^{+}-\mathbf{R}^{+}$where $\beta \mathbf{R}^{+}$is the Stone-Čech compactification of $\mathbf{R}^{+}$, the nonnegative reals [7].

The results of Seever and Wells are based, to a large extent, on the well-

Received by the editors July 30, 1973 and, in revised form, July 15, 1974.

AMS (MOS) subject classifications (1970). Primary 46A40.

Key words and phrases. Archimedean Riesz spaces, order dual, sequential convergence, principal projection property, weak compactness. 
known characterization of Grothendieck [8] of relatively weakly compact sets of Radon measures. Grothendieck's criterion has recently been extended to the setting of Riesz spaces by Fremlin [6] and Burkinshaw [4], and these results shall provide the major tool for our investigation, which will be centered on Riesz spaces with property I and Riesz spaces with the principal projection property such that the Boolean algebra of principal components of each positive element has property I. This latter class of Riesz spaces, for brevity, will be said to have property PI.

Throughout the paper, $L$ will denote an Archimedean Riesz space whose order dual $L^{\sim}$ is separating. By $L_{n}^{\sim}$ (resp. $L_{c}^{\sim}$ ) will be denoted the band of normal integrals (resp. integrals).on $L . I_{L}$ will denote the order ideal generated by $L$ in $L_{, n}^{\sim \sim}$. A sequence $\left\{f_{n}\right\}_{n \in \omega} \subset L$ will be called disjoint if $\left|f_{n}\right| \wedge\left|f_{m}\right|=0$ for all $n, m$. Here $\omega$ denotes the set of natural numbers. The principal result of the paper is the following (Theorem 4.5) and extends the HahnVitali-Saks theorem referred to above.

THEOREM. Let the Riesz space $L$ have property I or property PI. Let $\left\{\phi_{n}\right\}_{n \in \omega} \subset L^{\sim}$ and suppose that $\phi(f)=\lim _{n \rightarrow \infty} \phi_{n}(f)$ exists for each $f \in L$. Then

(a) $\sup _{n}\left|\phi_{n}\left(f_{j}\right)\right| \rightarrow 0$ as $j \rightarrow \infty$ for each order bounded disjoint sequence $\left\{f_{j}\right\} \subset L^{+}$.

(b) The linear form $\phi$ is order bounded, and $\phi(f)=\lim _{n \rightarrow \infty} \phi_{n}(f)$ holds for each $f \in I_{L}$.

(c) If $\left\{\phi_{n}\right\} \subset L_{c}^{\sim}\left(\right.$ resp. $\left.L_{n}^{\sim}\right)$, then $\phi \in L_{c}^{\sim}\left(\right.$ resp. $\left.L_{n}^{\sim}\right)$.

Theorem 4.7 extends the Nikodym boundedness theorem and states that if $L$ has property I or property PI, then a subset $A$ of the order dual $L^{\sim}$ is pointwise bounded on $L$ if and only if $A$ is uniformly bounded on each order interval of $L$. The main result of Andô [3] is extended in Theorem 4.8 and contains as an immediate corollary the well-known lemma of Phillips [5]. Finally the results of $\$ 5$ extend some of the work of Moore and Reber [12].

1. Preliminary results. The fundamental result on which the paper rests is the following extension of Grothendieck's characterization of relatively weakly compact sets of Radon measures.

THEOREM A. If $L$ is an Archimedean Riesz space, the following are equivalent for a subset $A \subset L^{\sim}$.

(i) $\sup \left\{\left|\phi\left(f_{n}-f_{m}\right)\right|: \phi \in A\right\} \rightarrow 0$ as $m, n \rightarrow \infty$ for each monotone order bounded sequence $\left\{f_{n}\right\} \subset L$. 
(ii) $\sup \left\{\left|\phi\left(f_{n}-f_{m}\right)\right|: \phi \in A\right\} \rightarrow 0$ as $m, n \rightarrow \infty$ for each monotone order bounded sequence $\left\{f_{n}\right\} \subset I_{L}$.

(iii) The solid hull of $A$ is relatively $\sigma\left(L^{\sim}, I_{L}\right)$ compact.

(iv) $A$ is $\sigma\left(L^{\sim}, L\right)$ bounded and $\sup \left\{\left|\phi\left(f_{n}\right)\right|: \phi \in A\right\} \rightarrow 0$ as $n \rightarrow \infty$ for each disjoint order bounded sequence $\left\{f_{n}\right\} \subset L^{+}$.

The equivalence of (i)-(iii) is proved by Burkinshaw [4] and derives from the work of Kaplan [9] which in turn stems from Amemiya [2]. The equivalence of (i)-(iii) with (iv) is due to Fremlin [6].

A subset $A \subset L^{\sim}$ will be called $|\sigma|\left(L^{\sim}, L\right)$ bounded if and only if $\sup \{|\phi|(e): \phi \in A\}<\infty$ for every $0 \leqslant e \in L$. We shall need the following result due to Fremlin [6] (see also [9]).

THEOREM B. If $L$ is an Archimedean Riesz space the following are equivalent for a subset $A \subset L^{\sim}$ :

(i) $A$ is $|\sigma|\left(L^{\sim}, L\right)$ bounded.

(ii) $A$ is $\sigma\left(L^{\sim}, L\right)$ bounded, and for each $0 \leqslant e \in L$,

$$
\sup _{n} \sup \left\{\left|\phi\left(f_{n}\right)\right|: \phi \in A\right\}<\infty
$$

for each disjoint sequence $\left\{f_{n}\right\} \subset[0, e]$.

We shall adopt the notation and terminology from the theory of Riesz spaces as presented in the papers [10] and monograph [11] of W. A. J. Luxemburg and A. C. Zaanen, and shall not in general refer explicitly to results from these sources.

It is a pleasure to thank O. Burkinshaw and D. H. Fremlin for making available their results prior to publication and for their comments on an earlier version of this paper.

2. Riesz spaces with property I. In this section we wish to gather some remarks concerning Riesz spaces with property I which are essentially known. The first is an analogue of Theorem 2.2 of [15].

Proposition 2.1. If the Riesz space $L$ has property $\mathrm{I}$, and $0 \leqslant \phi \in L^{\sim}$ is strictly positive on $L$, then $L$ is super Dedekind complete.

Proof. In view of Lemma 27.16 and Theorem 31.11 of [10] it is sufficient to prove that $L$ is Dedekind $\sigma$-complete. Let $0 \leqslant f_{n} \uparrow_{n} \leqslant f \in L$ and denote by $U$ the set of upper bounds of the sequence $\left\{f_{n}\right\}_{n \in \omega}$. Choose $\left\{g_{n}\right\}_{n \in \omega} \subset U$ such that $g_{n} \downarrow_{n}$ and $\inf _{n} \phi\left(g_{n}\right)=\inf \{\phi(g): g \in U\}$. Let $0 \leqslant$ $v \in L$ satisfy $f_{n} \leqslant v \leqslant g_{n}$ for $n \in \omega$. It is clear that $\phi(v)=\inf \{\phi(g): g \in U\}$. If $g$ is any member of $U$, then $v \wedge g \in U$ so $\phi(v)=\phi(v \wedge g)$. Thus 
$\phi(v-v \wedge g)=0$ and since $\phi$ is strictly positive on $L$, it follows that $v=v \wedge g$ and so $v=\sup _{n} f_{n}$.

Proposition 2.2. (a) Let $L, M$ be Riesz spaces, $h: L \rightarrow M$ a Riesz homomorphism of $L$ onto $M$. If $L$ has property I then $M$ has property I.

(b) If $L$ is a Riesz space with property $\mathrm{I}$, and if $J \subseteq L$ is an ideal, then $J$ has property $\mathrm{I}$.

Part (a) of the proposition is proved in [15, Theorem 2.1]. Part (b) of the proposition is a simple consequence of the definitions.

It is appropriate at this point to make an observation due to the referee and bring to the reader's attention the paper of Amemiya [1]. Let $\Phi \subset L^{+}$satisfy $0 \notin \Phi, u, v \in \Phi \Rightarrow u \wedge v \in \Phi$, and $u \in \Phi, w \geqslant u \Rightarrow w \in \Phi$. Denote by $J(\Phi)$ the collection of intervals $\{[0, u]: u \in \Phi\}$. Amemiya shows that if $L$ is a Dedekind complete Riesz space, then $L$ may be endowed with a topology which has Baire's property with respect to translates of the members of $J(\Phi)$. (A topological space $(X, \tau)$ is said to have Baire's property with respect to a family $A$ of subsets of $X$ if, whenever $\left\{F_{n}\right\}_{n \in \omega} \subset X$ is a sequence of closed sets such that $\bigcup_{n \in \omega} F_{n}$ contains a member of $A$, it follows that at least one of the sets $F_{n}$ already contains a member of $A$.) The fact that $L$ is assumed Dedekind complete is not essential and an inspection of Amemiya's proof shows immediately that the natural domain for his principle is that $L$ should have property I. As an application of his principle, Amemiya proves the following theorems.

THEOREM 2.3. If the Riesz space $L$ has property I, the following are equiv alent for a subset $A \subset L^{\sim}$.

(i) $A$ is $\sigma\left(L^{\sim}, L\right)$ bounded.

(ii) $A$ is $|\sigma|\left(L^{\sim}, L\right)$ bounded.

THEOREM 2.4. Let the Riesz space $L$ have property I. Then

(a) $L^{\sim}$ is $\sigma\left(L^{\sim}, L\right)$. sequentially complete.

(b) $L_{c}^{\sim}$ is $\sigma\left(L_{c}^{\sim}, L\right)$ sequentially complete.

(c) $L_{n}^{\sim}$ is $\sigma\left(L_{n}^{\sim}, L\right)$ sequentially complete.

For details, the reader is referred to the paper [1]. In $\S 4$ of the present paper, the above results will be proved by a sliding hump technique which is applicable to a much wider class of Riesz spaces other than those with property $I$.

3. Riesz spaces with the principal projection property. In this section a few minor results. will be presented which will make somewhat more transparent the relation of the results of $\S 4$ to the classical situation.

Proposition 3.1. Let the Riesz space $L$ have the principal projection prop- 
erty. If $\phi \in L^{\sim}, 0 \leqslant f \in L$ and $e$ is a principal component of $f$, then

$$
\begin{aligned}
|\phi|(e) & =\sup \{|\phi(h)|: 0 \leqslant|h| \leqslant e, h \in L\} \\
& =\sup \left\{\phi\left(e_{1}-e_{2}\right): e_{1}, e_{2} \text { are principal components of } e\right\} .
\end{aligned}
$$

Proof. The equality $|\phi|(e)=\sup \{|\phi(h)|: 0 \leqslant|h| \leqslant e\}$ is well known. It is clear that if $0 \leqslant e_{1}, e_{2} \in L$ satisfying $e_{1} \wedge e_{2}=0, e_{1}+e_{2}=e$, then $\phi\left(e_{1}-e_{2}\right) \leqslant|\phi|(e)$. Let $\epsilon>0$ be given and let $h \in L$ satisfy $0 \leqslant|h| \leqslant e$. By the Freudenthal spectral theorem [11], there exist disjoint principal components $\left\{e_{i}\right\}_{i=1}^{n}$ of $e$ and numbers $\left\{\alpha_{i}\right\}_{i=1}^{n}$ with $\left|\alpha_{i}\right| \leqslant 1$ such that $\left|h-\sum_{i=1}^{n} \alpha_{i} e_{i}\right|<$ $\epsilon e$. Thus $\left|\phi\left(h-\sum_{i=1}^{n} \alpha_{i} e_{i}\right)\right| \leqslant \epsilon|\phi|(e)$, from which it follows that

$$
\begin{aligned}
|\phi(h)|-\epsilon|\phi|(e) & \leqslant\left|\phi\left(\sum_{i=1}^{n} \alpha_{i} e_{i}\right)\right| \\
& \leqslant \sum_{i=1}^{n}\left|\alpha_{i}\right|\left|\phi\left(e_{i}\right)\right| \leqslant \sum_{i=1}^{n}\left|\phi\left(e_{i}\right)\right|=\phi\left(e^{\prime}-e^{\prime \prime}\right),
\end{aligned}
$$

where $e^{\prime}=\Sigma\left\{e_{i}: \phi\left(e_{i}\right) \geqslant 0\right\}$ and $e^{\prime \prime}=\Sigma\left\{e_{i}: \phi\left(e_{i}\right) \leqslant 0\right\}$. Since $e^{\prime} \wedge e^{\prime \prime}=0$ and $e^{\prime}+e^{\prime \prime}=e$, it follows that

$$
|\phi|(e) \leqslant \sup \left\{\phi\left(e_{1}-e_{2}\right): 0 \leqslant e_{1}, e_{2}, e_{1} \wedge e_{2}=0, e_{1}+e_{2}=e\right\}
$$

and the proof is complete.

Proposition 3.2. If the Riesz space $L$ has the principal projection property and $0 \leqslant e \in L$, the following are equivalent for a subset $A \subset L^{\sim}$.

(i) $\sup \left\{|\phi|\left(f_{n}\right): \phi \in A\right\} \rightarrow 0$ for each disjoint sequence $\left\{f_{n}\right\} \subset[0, e]$.

(ii) $\sup \left\{\left|\phi\left(e_{n}\right)\right|: \phi \in A\right\} \rightarrow 0$ for each disjoint sequence $\left\{e_{n}\right\}$ of principal components of $e$.

Proof. The implication (i) $\Rightarrow$ (ii) is obvious. If (i) does not hold, there exists $\epsilon>0$, a sequence $\left\{\phi_{n}\right\} \subset A$ and a disjoint sequence $\left\{f_{n}\right\} \subset[0, e]$ such that $\left|\phi_{n}\right|\left(f_{n}\right) \geqslant \epsilon$ for $n \in \omega$. Denote by $f_{n}^{\prime}$ the component of $e$ in the principal band generated in $L$ by $f_{n}$, for $n \in \omega$, and note that the sequence $\left\{f_{n}^{\prime}\right\}$ is disjoint and that $f_{n}^{\prime} \geqslant f_{n}$ for $n \in \omega$. Since each principal component of a principal component of $e$ is again a principal component of $e$, it follows readily from Proposition 3.1 that there exist principal components $\left\{e_{n}\right\}_{n \in \omega}$ of $e$ with $e_{n} \leqslant f_{n}^{\prime}$ and $\left|\phi_{n}\left(e_{n}\right)\right| \geqslant \epsilon / 2$ for $n \in \omega$, so (ii) does not hold and the proof is complete.

PROPOSITION 3.3. If the Riesz space $L$ has the principal projection property, $0 \leqslant e \in L$, and $A \subset L^{\sim}$ is $\sigma\left(L^{\sim}, L\right)$ bounded, the following statements are equivalent:

(i) $\sup \left\{\left|\phi\left(f_{n}-f_{m}\right)\right|: \phi \in A\right\} \rightarrow 0$ as $n, m \rightarrow \infty$ for each monotone sequence $\left\{f_{n}\right\} \subset[0, e]$. 
(ii) $\sup \left\{\left|\phi\left(e_{n}-e_{m}\right)\right|: \phi \in A\right\} \rightarrow 0$ as $n, m \rightarrow \infty$ for each monotone sequence $\left\{e_{n}\right\}$ of principal components of $e$.

(iii) $\sup \left\{|\phi|\left(e_{n}\right): \phi \in A\right\} \rightarrow 0$ as $n \rightarrow \infty$ for each disjoint sequence $\left\{e_{n}\right\}$ of principal components of $e$.

Proof. The implication (i) $\Rightarrow$ (ii) is obvious. That (ii) $\Rightarrow$ (iii) follows from Proposition 3.2 and the observation that if $\left\{e_{j}^{\prime}\right\}$ is a disjoint sequence of principal components of $e$, then $e_{n}=\sum_{j=1}^{n} e_{j}^{\prime}$ is a monotone sequence of principal components of $e$. The implication (iii) $\Rightarrow$ (i) follows from Theorem $A$ and Proposition 3.2.

Proposition 3.4. Let the Riesz space $L$ have the principal projection property, $0 \leqslant e \in L$ and assume that the Boolean algebra of principal components of $e$ is o-complete. Let $A \subset L_{c}^{\tilde{c}}$ be $\sigma\left(L_{c}^{\tilde{c}}, L\right)$ bounded. Each of the following statements is equivalent to each of the statements (i)-(iii) of Proposition 3.3:

(iv) For each sequence $\left\{e_{n}\right\}$ of principal components of $e$,

$$
e_{n} \downarrow_{n} 0 \Rightarrow \sup \left\{\left|\phi\left(e_{n}\right)\right|: \phi \in A\right\} \rightarrow 0 \text {. }
$$

(v) For each sequence $\left\{f_{n}\right\} \subset[0, e]$,

$$
e \geqslant f_{n} \downarrow 0 \Rightarrow \inf _{n} \sup \left\{|\phi|\left(f_{n}\right): \phi \in A\right\}=0 .
$$

The proof is similar to that of Proposition 3.5 below and will be omitted.

Proposition 3.5. Let the Riesz space $L$ have the principal projection property, $0 \leqslant e \in L$ and assume that the Boolean algebra of principal components of $e$ is o-complete. Let $A \subset L_{n}^{\tilde{n}}$ be $\sigma\left(L_{n}^{\tilde{n}}, L\right)$ bounded. Each of the following statements is equivalent to each of the statements (i) - (iii) of Proposition 3.3.

(vi) For each system of principal components $\left\{e_{\tau}\right\}$ of $e$

$$
e_{\tau} \downarrow_{\tau} 0 \Rightarrow \sup \left\{\left|\phi\left(e_{\tau}\right)\right|: \phi \in A\right\} \rightarrow 0 \text {. }
$$

(vii) For each system $\left\{f_{\tau}\right\} \subset[0, e]$

$$
e \geqslant f_{\tau} \downarrow_{\tau} 0 \Rightarrow \inf _{\tau} \sup \left\{|\phi|\left(f_{\tau}\right): \phi \in A\right\}=0 .
$$

Proof. That (vii) $\Rightarrow$ (vi) is clear. To show that (vi) $\Rightarrow$ (ii) of Proposition 3.3, let $\left\{e_{n}\right\}$ be a monotone sequence of principal components of $e$. Suppose for definiteness that $e_{n} \downarrow_{n}$. Let $e=\bigwedge_{n} e_{n}$ and note that $e_{n} \downarrow_{n} e$ holds in $L$. By (vi), $\sup \left\{\left|\phi\left(e_{n}-e\right)\right|: \phi \in A\right\} \rightarrow 0$ as $n \rightarrow \infty$, from which (ii) clearly follows.

Now suppose that (i) of Proposition 3.3 is satisfied for $A$. Proposition 3.3(iii) then implies that (i) is also satisfied for the solid hull of $A$. Let $e \geqslant$ 
$f_{\tau} \downarrow_{\tau} 0$ and assume that $\inf _{\tau} \sup \left\{|\phi|\left(f_{\tau}\right): \phi \in A\right\}>0$. It is easily verified that there is a number $\epsilon>0$, a subsequence $\left\{f_{\tau_{n}}\right\} \subset\left\{f_{\tau}\right\}$ with $f_{\tau_{n}} \downarrow_{n}$ and a sequence $\left\{\phi_{n}\right\} \subset A$ such that $\left|\phi_{n}\right|\left(f_{\tau_{k}}\right) \rightarrow 0$ as $k \rightarrow \infty$ for each $n \in \omega$ and $\left|\phi_{n}\right|\left(f_{\tau_{n}}\right) \geqslant \epsilon>0$ for $n \in \omega$. Hence, it follows from (i) that $\sup _{n}\left|\phi_{n}\right|\left(f_{\tau_{k}}\right) \rightarrow 0$ as $k \rightarrow \infty$ and this is clearly a contradiction. Thus (i) of Proposition 3.3 implies (vii) and the chain of implications is complete.

\section{Main results.}

LEMMA 4.1. Let the Riesz space $L$ have property $\mathrm{I}, 0 \leqslant e \in L$ and $\left\{e_{n}\right\}_{n \in \omega} \subset[0, e]$ be a disjoint sequence. If $f \in[0, e]$ and $f \wedge e_{n}=0$ for each $n \in \omega$, there exists $h \in[0, e]$ with $f \wedge h=0$ and $\left\{e_{n}\right\}_{n \in \omega} \subset[0, h]$.

Proof. Observe that $e_{1}+\cdots+e_{n} \uparrow_{n}, e-n f \wedge e_{n} \downarrow_{n}$ and $e_{1}+\cdots+$ $e_{n} \leqslant e-m f \wedge e=(e-m f)^{+}$holds for each $n, m \in \omega$. By property $\mathrm{I}$, there exists $h \in L$ with $e_{1}+\cdots+e_{n} \leqslant h \leqslant(e-n f)^{+}$for all $n \in \omega$. Observe now that $f \wedge(e-n f)^{+} \downarrow_{n} 0$. In fact, if $0 \leqslant g \leqslant f \wedge(e-n f)^{+}$holds for all $n \in$ $\omega$, it follows from $g \leqslant f$ that $g \wedge(f-e / n)^{+} \uparrow_{n} g$. Since $g \leqslant(e-n f)^{+}$implies $g \wedge(f-e / n)^{+}=g \wedge(e-n f)^{+} \wedge(f-e / n)^{+}=0$, it follows that $g=0$. In particular then, $0 \leqslant f \wedge h \leqslant f \wedge(e-n f)^{+}$for $n \in \omega$ implies $f \wedge h=0$.

Definition 4.2. The Riesz space $L$ will be said to have property PI if $L$ has the principal projection property and for each $0 \leqslant e \in L$, the Boolean aigebra of principal components of $e$ has property $\mathrm{I}$.

It is easily seen that any Riesz space with the projection property, or which is Dedekind $\sigma$-complete has property PI. Of course, the prime example of a Riesz space with property PI we have in mind is the Riesz space of real, finitelyvalued functions defined on a point set $X$ and measurable with respect to a Boolean algebra of subsets of $X$ which has property $\mathrm{I}$.

LEMma 4.3. Let the Riesz space $L$ have property I or property PI. If $0 \leqslant e \in L$, and $\left\{e_{n}\right\}_{n \in \omega},\left\{e_{n}^{\prime}\right\}_{n \in \omega} \subset[0, e]$ are disjoint sequences with $e_{n} \wedge$ $e_{m}^{\prime}=0$ for all $n, m$, there exist $h, h^{\prime} \in[0, e]$ with $h \wedge h^{\prime}=0,\left\{e_{n}\right\}_{n \in \omega} \subset$ $[0, h]$ and $\left\{e_{n}^{\prime}\right\}_{n \in \omega} \subset\left[0, h^{\prime}\right]$. If $L$ has property $\mathrm{PI}$, then $h, h^{\prime}$ can be taken to be principal components of $e$.

Proof. Assume first that $L$ has property I. By Lemma 4.1, there exists a sequence $\left\{h_{n}^{\prime \prime}\right\} \subset[0, e]$ such that $h_{n}^{\prime \prime} \wedge e_{n}^{\prime}=0$ and $\left\{e_{j}\right\}_{j \in \omega} \subset\left[0, h_{n}^{\prime \prime}\right]$ for each $n \in \omega$. Let $h_{n}^{\prime}=h_{1}^{\prime \prime} \wedge \cdots \wedge h_{n}^{\prime \prime}$ and observe that $h_{n}^{\prime} \wedge\left(e_{1}^{\prime}+\cdots+\right.$ $\left.e_{n}^{\prime}\right)=0$ for each $n \in \omega$. By property $\mathrm{I}$, there exists $h \in[0, e]$ such that $\left\{e_{j}\right\}_{j \in \omega} \subset[0, h] \subset\left[0, h_{n}^{\prime}\right]$ for each $n \in \omega$. It is clear that $h \wedge e_{j}^{\prime}=0$ for $j \in \omega$. Again by Lemma 4.1, there exists $h^{\prime} \in[0, e]$ with $h \wedge h^{\prime}=0$ and $\left\{e_{j}^{\prime}\right\}_{j \in \omega} \subset\left[0, h^{\prime}\right]$. 
Assume now that $L$ has property PI. Denote by $f_{n}, f_{n}^{\prime}$ respectively the components of $e$ in the principal bands generated in $L$ by $e_{n}, e_{n}^{\prime}$. Observe that $\left\{f_{n}\right\}_{n \in \omega},\left\{f_{n}^{\prime}\right\}_{n \in \omega}$ are again disjoint sequences which are mutually disjoint. Note that if $f^{\prime}$ is a principal component of $e$ such that $f^{\prime} \wedge f_{n}=0$ for each $n \in \omega$, then the principal component $f=e-f^{\prime}$ satisfies $f \wedge f^{\prime}=0$ and $\left\{f_{n}\right\}_{n \in \omega} \subset[0, f]$. The proof now proceeds exactly as in the case that $L$ has property $\mathrm{I}$, by working in the Boolean algebra of principal components of $e$ rather than in $L$ itself. The details are omitted.

Let now $\left\{N_{i}\right\}_{i \in \omega}$ be a partition of $\omega$ into a sequence of mutually disjoint infinite subsets $N_{i}$. If $L$ has property I or property PI, $0 \leqslant e \in L$ and $\left\{e_{n}\right\}_{n \in \omega} \subset[0, e]$ is a disjoint sequence, it follows from Lemma 4.3 that there exists a disjoint sequence $\left\{h_{i}\right\}_{i \in \omega} \subset[0, e]$ such that $\left\{e_{j}\right\}_{j \in N_{i}} \subset\left[0, h_{i}\right]$ for each $i \in \omega$. Let now $\phi \in L^{\sim}$ and $\epsilon>0$ be given. Since $\Sigma_{i=1}^{\infty}|\phi|\left(h_{i}\right) \leqslant$ $|\phi|(e)$, it follows that there exists an index $i_{0}$ such that $|\phi|\left(h_{i}\right)<\epsilon$ for all $i \geqslant$ $i_{0}$. These remarks constitute the proof of

LEMma 4.4. Let $L$ have property I or property $\mathrm{PI}, 0 \leqslant e \in L$ and let $\left\{e_{n}\right\}_{n \in \omega} \subset[0, e]$ be a disjoint sequence. If $\phi \in L^{\sim}$ and $\epsilon>0$ is given, there exists an infinite subset $N \subset \omega$ and $h_{N} \in[0, e]$ such that $\left\{e_{j}: j \in N\right\} \subset\left[0, h_{N}\right]$ and $|\phi|\left(h_{N}\right)<\epsilon$. If $L$ has property $\mathrm{PI}$, then $h_{N}$ may be taken to be a principal component of $e$.

We come now to the principal result of this section.

THEOREM 4.5. Let the Riesz space $L$ have property I or property PI. Let $\left\{\phi_{n}\right\}_{n \in \omega} \subset L^{\sim}$ and suppose that $\phi(f)=\lim _{n \rightarrow \infty} \phi_{n}(f)$ exists for each $f \in L$. Then

(a) For each $0 \leqslant e \in L, \sup _{n}\left|\phi_{n}\left(e_{j}\right)\right| \rightarrow 0$ as $j \rightarrow \infty$ for every disjoint sequence $\left\{e_{j}\right\}_{j \in \omega} \subset[0, e]$.

(b) $\phi \in L^{\sim}$ and $\phi(f)=\lim _{n \rightarrow \infty} \phi_{n}(f)$ holds for all $f \in I_{L}$.

(c) If $\left\{\phi_{n}\right\}_{n \in \omega} \subset L_{c}^{\sim}\left(\right.$ resp. $\left.L_{n}^{\sim}\right)$, then $\phi \in L_{c}^{\sim}$ (resp. $\left.L_{n}^{\sim}\right)$.

Proof. Assume first that $L$ has property I and that (a) is not satisfied. Without loss of generality, assume that there exists $\epsilon>0,0 \leqslant e \in L$, a disjoint sequence $\left\{e_{j}\right\} \subset[0, e]$ such that $\left|\phi_{n}\left(e_{n}\right)\right|>\epsilon$ for all $n \in \omega$. Let $n_{1}=1$ and find inductively a sequence of positive integers $n_{k} \uparrow_{k}$ such that $\left|\phi_{n_{k}}\right|\left(e_{n_{k+1}}\right)<$ $\epsilon / 2$. This is possible since $\Sigma_{k=1}^{\infty}\left|\phi_{j}\right|\left(e_{k}\right) \leqslant\left|\phi_{j}\right|(e)$ holds for each $j \in \omega$. Observe that

$$
\left|\left(\phi_{n_{k+1}}-\phi_{n_{k}}\right)\left(e_{n_{k+1}}\right)\right| \geqslant\left|\phi_{n_{k+1}}\left(e_{n_{k+1}}\right)\right|-\left|\phi_{n_{k}}\right|\left(e_{n_{k+1}}\right)>\epsilon / 2 .
$$

By suitable relabelling, it may therefore be assumed that $\left|\phi_{n}\left(e_{n}\right)\right|>\epsilon$ for all $n \in \omega$ and, in addition, $\lim _{n \rightarrow \infty} \phi_{n}(f)=0$ for each $f \in L$. By Lemma 4.3, 
there exists an infinite subset $N_{1} \subset \omega, h_{N_{1}} \in[0, e]$ such that $h_{N_{1}} \wedge e_{1}=0$, $\left|\phi_{1}\right|\left(h_{N_{1}}\right)<\epsilon / 3$ and $\left\{e_{j}: j \in N_{1}\right\} \subset\left[0, h_{N_{1}}\right]$. Since $\phi_{n}\left(e_{1}\right) \rightarrow 0$ as $n \rightarrow \infty$, it may be assumed further that $\left|\phi_{j}\left(e_{1}\right)\right|<\epsilon / 3$ for all $j \in N_{1}$. Letting $n_{1}=1$, define inductively integers $n_{k} \uparrow_{k}$, infinite subsets $\left\{N_{k}\right\} \downarrow_{k} \subset \omega$ and $h_{N_{k}} \downarrow_{k} \subset$ $[0, e]$ with the following properties:

(a) $N_{k} \subset N_{k-1}, n_{k} \in N_{k-1}, k=2,3, \cdots$.

(b) $h_{N_{k}} \wedge e_{n_{j}}=0,1 \leqslant j \leqslant k$, and $\left\{e_{j}: j \in N_{k}\right\} \subset\left[0, h_{N_{k}}\right]$.

(c) $\sum_{i=1}^{k}\left|\phi_{j}\left(e_{n_{i}}\right)\right|<\epsilon / 3$ for all $j \in N_{k}$.

(d) $\left|\phi_{n_{k}}\right|\left(h_{N_{k}}\right)<\epsilon / 3$.

Now note that

and

$$
e_{n_{1}} \vee \cdots \vee e_{n_{k}} \vee h_{N_{k}} \downarrow_{k}, \quad e_{n_{1}} \vee \cdots \vee e_{n_{k}} \uparrow_{k}
$$

$$
e_{n_{1}} \vee \cdots \vee e_{n_{k}} \leqslant e_{n_{1}} \vee \cdots \vee e_{n_{k}} \vee h_{N_{k}}
$$

hold for each $k \in \omega$. By property I, there exists $h \in[0, e]$ such that

$$
e_{n_{1}} \vee \ldots \vee e_{n_{k}} \leqslant h \leqslant e_{n_{1}} \vee \cdots \vee e_{n_{k}} \vee h_{N_{k}} \text { for all } k \in \omega .
$$

Note that $0 \leqslant h-e_{n_{1}} \vee \cdots \vee e_{n_{k}} \leqslant h_{N_{k}}$ and so

Thus

$$
\left|\phi_{n_{k}}\right|\left(h-e_{n_{1}} \vee \cdots \vee e_{n_{k}}\right) \leqslant\left|\phi_{n_{k}}\right|\left(f_{N_{k}}\right)<\epsilon / 3 \text {. }
$$

$$
\begin{aligned}
\left|\phi_{n_{k}}(h)\right| & \geqslant\left|\phi_{n_{k}}\left(e_{n_{1}}+\cdots+e_{n_{k}}\right)\right|-\epsilon / 3 \\
& \geqslant\left|\phi_{n_{k}}\left(e_{n_{k}}\right)\right|-\sum_{i=1}^{k-1}\left|\phi_{n_{k}}\left(e_{n_{i}}\right)\right|-\epsilon / 3>\epsilon / 3
\end{aligned}
$$

which contradicts the fact that $\phi_{n_{k}}(h) \rightarrow 0$ as $k \rightarrow \infty$, and so (a) is proved.

It is now an immediate consequence of Theorem B that $\left\{\phi_{n}\right\}$ is $|\sigma|\left(L^{\sim}, L\right)$ bounded so it is immediate that the linear functional $\phi$, defined by setting $\phi(f)=$ $\lim _{n \rightarrow \infty} \phi_{n}(f)$ for $f \in L$, is in fact order bounded. That $\phi(f)=\lim _{n \rightarrow \infty} \phi_{n}(f)$ holds for all $f \in I_{L}$ follows immediately from the fact that $\left\{\phi_{n}\right\} \subset L^{\sim}$ is relatively $\sigma\left(L^{\sim}, I_{L}\right)$ compact by Theorem A and so (b) is proved.

Suppose now that $\left\{\phi_{n}\right\} \subset L_{c}^{\sim}$ and that $0 \leqslant f_{n} \downarrow_{n} 0$ holds for the sequence $\left\{f_{n}\right\} \subset L$. Observe that by (i) of Theorem $\mathrm{A}$ it follows that $\inf _{k} \sup _{n}\left|\phi_{n}\right|\left(f_{k}\right)$ $=0$. This implies immediately that $\phi \in L_{c}^{\sim}$ and a similar calculation holds for the case that $\left\{\phi_{n}\right\} \subset L_{n}^{\sim}$.

If the Riesz space $L$ has property PI rather than property $\mathrm{I}$, the proof of the theorem is virtually identical. Via Proposition 3.2, it is only necessary to work with disjoint sequences of principal components, rather than with all order bounded disjoint sequences.

The above Theorem 4.5 extends the classical theorem of Vitali-Hahn-Saks [14] alluded to in the Introduction. It is now an easy matter to extend the boundedness theorem of 0 . Nikodym [13]. 
THEOREM 4.6. Let the Riesz space $L$ have property I or property PI. The following statements are equivalent for a subset $A \subset L^{\sim}$.

(i) $A$ is $\sigma\left(L^{\sim}, L\right)$ bounded.

(ii) $A$ is $|\sigma|\left(L^{\sim}, L\right)$ bounded.

Proof. It is clearly only necessary to prove that (i) $\Rightarrow$ (ii). Suppose then that $A \subset L^{\sim}$ is $\sigma\left(L^{\sim}, L\right)$ bounded but not $|\sigma|\left(L^{\sim}, L\right)$ bounded. By Theorem $B$ and a suitable change of notation, there exists $0 \leqslant e \in L,\left\{\phi_{n}\right\} \subset A$, a disjoint sequence $\left\{e_{n}\right\}_{n \in \omega} \subset[0, e]$ such that $\left|\phi_{n}\left(e_{n}\right)\right|>n^{2}$ for all $n \in \omega$. Let $\psi_{n}=\phi_{n} / n$ and observe that $\psi_{n}(f) \rightarrow 0$ for each $f \in L$ and $\left|\psi_{n}\left(e_{n}\right)\right|>n$. This however contradicts (a) of Theorem 4.6 and the proof is complete.

THEOREM 4.7. Let the Riesz space $L$ have property I or property PI. Let $B \subset L^{\sim}$ be a band and $P: L^{\sim} \rightarrow B$ the corresponding projection. If $\left\{\phi_{n}\right\} \subset L^{\sim}$ and $\phi_{n} \rightarrow 0 \sigma\left(L^{\sim}, L\right)$, then also $P \phi_{n} \rightarrow 0 \sigma\left(L^{\sim}, L\right)$. If $B$ is discrete, then $P\left|\phi_{n}\right| \rightarrow 0 \sigma\left(L^{\sim}, L\right)$.

Proof. Denote by $Q$ the projection of $I_{L}$ onto the carrier band of $B$ in $I_{L}$. By Theorem 27.12 of [10], note that $(P \phi)(f)=\phi(Q f)$ for $\phi \in L^{\sim}, f \in I_{L}$. It follows from Theorem 4.5 that for $f \in I_{L},\left(P \phi_{n}\right)(f)=\phi_{n}(Q f) \rightarrow 0$ as $n \rightarrow \infty$.

Denote by $\left\{\psi_{i}\right\}_{i \in I}$ the set of atoms of $B$, and for each finite subset $\tau \subset I$ let $P_{\tau}=\Sigma_{i \in \tau} P_{\psi i}$, where $P_{\psi}$ denotes the projection of $L^{\sim}$ onto the principal band generated by $\psi \in L^{\sim}$. Observe $P_{\tau} \uparrow_{\tau} P$ and let $Q_{\tau}$ be the projection of $I_{L}$ onto the carrier band in $I_{L}$ of the band $P_{\tau} L^{\sim}$. Let $\epsilon>0$ be given and $0 \leqslant f \in I_{L}$. Since $Q_{\tau} f \uparrow_{\tau} Q f$, it follows from Theorem $A$ and Theorem 4.5 that there exists an index $\tau_{0}$ such that $\sup _{n \in \omega}\left\{\left|\phi_{n}\right|\left(Q f-Q_{\tau_{0}} f\right)\right\}<\epsilon$. Note that $\left(P_{\psi}|\phi|\right)(f)=\left|\left(P_{\psi} \phi\right)(f)\right|$ holds for each atom $\psi$, each $\phi \in L^{\sim}$, and $0 \leqslant f \in I_{L}$. Thus

$$
\begin{aligned}
\left(P\left|\phi_{n}\right|\right)(f) & =\left(P\left|\phi_{n}\right|\right)(Q f) \leqslant \sup _{n}\left\{P\left|\phi_{n}\right|\left(Q f-Q_{\tau_{0}} f\right)\right\}+\left(P\left|\phi_{n}\right|\right)\left(Q_{\tau_{0}} f\right) \\
& \leqslant \epsilon+\sum_{i \in \tau_{0}}\left(P_{\psi_{i}}\left|\phi_{n}\right|\right)(f)=\epsilon+\sum_{i \in \tau_{0}}\left|\left(P_{\psi_{i}} \phi_{n}\right)(f)\right| .
\end{aligned}
$$

It follows that $\overline{\lim }_{n \rightarrow \infty}\left(P\left|\phi_{n}\right|\right)(f) \leqslant \epsilon$ and the theorem is proved.

Denote by b.a. $\left(2^{\omega}\right)$ (resp. c.a. $\left(2^{\omega}\right)$ ) the Riesz space of all real-valued, bounded, additive (resp. countably additive) measures on the Boolean algebra of all subsets of $\omega$.

Corollary 4.8 (Phillips' lemma [5]). Let $\left\{\mu_{n}\right\}_{n \in \omega} \subset$ b.a. $\left(2^{\omega}\right)$ and suppose $\mu_{n}(E) \rightarrow 0$ as $n \rightarrow \infty$ for each $E \in 2^{\omega}$. Then $\Sigma_{k=1}^{\infty}\left|\mu_{n}(\{k\})\right| \rightarrow 0$. as $n \rightarrow \infty$. 
Proof. Identify each $\mu \in$ b.a. $\left(2^{\omega}\right)$ with its linear extension to an element of the order dual of the Riesz space of finitely-valued real-valued sequences and note that this latter space has certainly property PI. Let $P$ denote the band projection of b.a. $\left(2^{\omega}\right)$ onto c.a. $\left(2^{\omega}\right)$. It is well known that if $\mu \in$ b.a. $\left(2^{\omega}\right)$ and $E \in 2^{\omega}$, then $P|\mu|(E)=\Sigma_{i \in E}|\mu(\{i\})|$. The corollary now follows immediately from Theorem 4.7.

\section{Some applications to locally convex Riesz spaces.}

THEOREM 5.1. Let the Riesz space $L$ have the principal projection proper$t y$, and assume that for each $0 \leqslant e \in L$, the Boolean algebra of principal components of $e$ is $\sigma$-complete. The following statements are equivalent for a subset $A \subset L_{c}^{\sim}:$

(i) $A$ is $\sigma\left(L_{c}^{\sim}, L\right)$ bounded and $f_{n} \downarrow_{n} 0$ in $L \Rightarrow \inf _{n} \sup _{\phi \in A}\left\{|\phi|\left(f_{n}\right)\right\}=0$.

(ii) $A$ is $\sigma\left(L_{c}^{\sim}, L\right)$ relatively compact.

(iii) $A$ is $\sigma\left(L_{c}^{\sim}, I_{L}\right)$ relatively compact.

Proof. Note first that if it follows from Theorem A and Proposition 3.4, that $A \subset L_{c}^{\sim}$ is $\sigma\left(L_{c}^{\sim}, I_{L}\right)$ relatively compact if and only if $A$ is $\sigma\left(L^{\sim}, I_{L}\right)$ relatively compact. From this remark, it is clear that (i) $\Leftrightarrow$ (iii) $\Rightarrow$ (ii). Thus it is only necessary to prove the implication (ii) $\Rightarrow$ (iii) and to this end assume that $A$ is $\sigma\left(L_{c}^{\sim}, L\right)$ relatively compact but that (iv) of Theorem A is not satisfied. Without loss of generality it may be assumed via Proposition 3.2 that there exists $\epsilon>0,0 \leqslant e \in L$ a sequence $\left\{\phi_{n}\right\} \subset A$ and a disjoint sequence $\left\{e_{n}\right\}_{n \in \omega}$ of principal components of $e$ such that $\left|\phi_{j}\right|\left(e_{n}\right)<\epsilon$ for $j<n$ and $\left|\phi_{n}\left(e_{n}\right)\right| \geqslant 2 \epsilon$ for $n \in \omega$. By a diagonal process, it may be assumed further that $\lim _{n \rightarrow \infty} \phi_{n}\left(e_{k}\right)$ exists for $k \in \omega$. Let $\psi_{n}=\phi_{n}-\phi_{n-1}$ so that $\left|\psi_{n}\left(e_{n}\right)\right| \geqslant \epsilon$ for $n \in \omega$ and $\psi_{n}\left(e_{k}\right) \rightarrow 0$ as $n \rightarrow \infty$ for $k \in \omega$. It follows from Phillips' lemma that there exist subsequences $\left\{n_{j}\right\},\left\{m_{j}\right\}$ of $\omega$ and a number $\beta>0$ such that $\left|\psi_{m_{k}}\left(\Sigma_{j} e_{n_{j}}\right)\right|$ $\geqslant \beta>0$ for each $k \in \omega$. If $\psi$ is any $\sigma\left(L_{c}^{\sim}, L\right)$ accumulation point of $\left\{\psi_{m_{k}}\right\}$, then $\left|\psi\left(\Sigma_{j} e_{n_{j}}\right)\right| \geqslant \beta>0$ and for each integer $N, \psi\left(\sum_{j=1}^{N} e_{n_{j}}\right)=0$. However, since $\psi \in L_{c}^{\sim}, \psi\left(\sum_{j=1}^{\infty} e_{n_{j}}\right)=\lim _{N \rightarrow \infty} \psi\left(\sum_{j=1}^{N} e_{n_{j}}\right)=0$. This is a contradiction and so the proof is complete.

A similar result for normal integrals is valid, and this we state without proof.

THEOREM 5.2. Under the assumptions of Theorem 5.1, the following statements are equivalent for a subset $A \subset L_{n}^{\sim}$ :

(i) $A$ is $\sigma\left(L_{n}^{\sim}, L\right)$ bounded and $f_{\tau} \downarrow_{\tau} 0$ in $L \Rightarrow \inf _{\tau} \sup _{\phi \in A}|\phi|\left(f_{\tau}\right)=0$.

(ii) $A$ is $\sigma\left(L_{n}^{\sim}, L\right)$ relatively compact.

(iii) $A$ is $\sigma\left(L_{n}^{\sim}, I_{L}\right)$ relatively compact. 
The assumptions of Theorems 5.1, 5.2 are satisfied if either $L$ is Dedekind $\sigma$-complete or has the projection property. The following result extends Theorems $3.5,3.8$ of [12].

THEOREM 5.3. Let $L$ be a Riesz space with the principal projection property and for each $0 \leqslant e \in L$, assume that the Boolean algebra of principal components of $e$ is $\sigma$-complete. Suppose $L_{n}^{\sim}$ distinguishes the points of $L$ and let $D$ be the Dedekind completion of $L$. For a subset $A \subset L_{n}^{\sim}$, the following are equivalent:

(i) $A$ is $\sigma\left(L_{n}^{\sim}, L\right)$ relatively compact.

(ii) $A$ is $\sigma\left(L_{n}^{\sim}, D\right)$ relatively compact.

(iii) $A$ is $\sigma\left(L_{n}^{\sim}, L\right)$ bounded and $f_{\tau} \downarrow_{\tau} 0 \Rightarrow \inf _{\tau} \sup \left\{|\phi|\left(f_{\tau}\right): \phi \in A\right\}=0$. In particular, the Mackey topology $\tau\left(L, L_{n}^{\sim}\right)$ is a locally convex Riesz topology.

Proof. The equivalence of (i) and (iii) follows from Theorem 5.2 above. As is noted in [12], (iii) holds with $L$ replaced by $D$. Since $D_{n}^{\sim}=L_{n}^{\sim}$ the equivalence of (ii) and (iii) again follows from Theorem 5.2.

\section{REFERENCES}

1. I. Amemiya, On a topological method in semi-ordered linear spaces, Proc. Japan. Acad. 27 (1951), 138-140. MR 14, 59.

2. - On ordered topological linear spaces, Proc. Internat. Sympos. Linear Spaces (Jerusalem, 1961), Jerusalem Academic Press, Jerusalem; Pergamon, Oxford, 1961, pp. 1423. MR 25 \#2411.

3. T. Andô, Convergent sequences of finitely additive measures, Pacific J. Math. 11 (1961), 395-404. MR 25 \#1255.

4. O. Burkinshaw, Weak compactness in the order dual of an Archimedean Riesz space, Thesis, Purdue University, Lafayette, Ind., 1972.

5. M. M. Day, Normed linear spaces, Springer-Verlag, Berlin, 1958. MR 20 \#1187.

6. D. H. Fremlin, Topological Riesz spaces and measure theory, Cambridge Univ. Press, New York, 1974.

7. L. Gillman and M. Jerison, Rings of continuous functions, University Ser. in Higher Math., Van Nostrand, Princeton, N. J., 1960. MR 22 \#6994.

8. A. Grothendieck, Sur les applications linéaires faiblement compactes d'espaces du type $C(K)$, Canad. J. Math. 5 (1953), 129-173. MR 15, 438.

9. S. Kaplan, On weak compactness in the space of Radon measures, J. Functional Analysis 5 (1970), 259-298. MR 41 \#5932.

10. W. A. J. Luxemburg and A. C. Zaanen, Notes on Banach function spaces VIII-X, Nederl. Akad. Wetensch. Proc. Ser. A 67 = Indag. Math. 26 (1964), 104-119, 360-376, 493-506. MR 28 \#5324b; 30 \#3381a, b.

11. - Riesz spaces. Vol. I, North-Holland, Amsterdam, 1971.

12. L. C. Moore, Jr. and James C. Reber, Mackey topologies which are locally convex Riesz topologies, Duke Math. J. 39 (1972), 105-119. MR 45 \#4113. 
13. O. Nikodym, Sur les familles bornées de fonctions parfaitement additives d'ensemble abstrait, C. R. Acad. Sci. Paris 192 (1931), 727-728.

14. S. Saks, On some functionals, Trans. Amer. Math. Soc. 35 (1933), 549-556.

15. G. L. Seever, Measures on F-spaces, Trans. Amer. Math. Soc. 133 (1968), 267280. MR $37 \# 1976$.

16. B. B. Wells, Jr., Weak compactness of measures, Proc. Amer. Math. Soc. 20 (1969), 124-130. MR 38 \#6343.

17. A. C. Zaanen, Integration, rev. ed., North-Holland, Amsterdam; Interscience, New York, 1967. MR $36 \# 5286$.

DEPARTMENT OF MATHEMATICS, FLINDERS UNIVERSITY OF SOUTH AUSTRALIA, BEDFORD PARK, SOUTH AUSTRALIA 5042, AUSTRALIA 